\title{
Making microbeams and nanobeams by channeling in microstructures and nanostructures
}

\author{
S. Bellucci, ${ }^{1}$ V. M. Biryukov, ${ }^{2, *}$ Yu. A. Chesnokov, ${ }^{2}$ V. Guidi, ${ }^{3}$ and W. Scandale ${ }^{4}$ \\ ${ }^{1}$ INFN, Laboratori Nazionali di Frascati, P.O. Box 13, 00044 Frascati, Italy \\ ${ }^{2}$ Institute for High Energy Physics, 142281 Protvino, Russia \\ ${ }^{3}$ Department of Physics and INFN, Via Paradiso 12, I-44100 Ferrara, Italy \\ ${ }^{4}$ CERN, Geneva, Switzerland \\ (Received 7 October 2002; published 17 March 2003)
}

\begin{abstract}
A particle beam of very small cross section is useful in many accelerator applications including biological and medical ones. We show the capability of the channeling technique using a micron-sized structure on a surface of a single crystal, or using a nanotube, to produce a beam of a cross section down to one square micrometer (or nanometer). The channeled beam can be deflected and thus well separated in angle and space from the primary and scattered particles. Monte Carlo simulation is done to evaluate the characteristics of a channeled microbeam. Emittances down to $0.001 \mathrm{~nm} \mathrm{rad}$, and flux up to $10^{6} \mu \mathrm{m}^{2}$ per second, can be achieved for protons and ions.
\end{abstract}

DOI: 10.1103/PhysRevSTAB.6.033502

\section{INTRODUCTION}

Bent crystals have efficiently channeled particle beams [1] in the energy range from $3 \mathrm{MeV}$ [2] to $900 \mathrm{GeV}$ [3]. Today, crystals are largely used for extraction of $70 \mathrm{GeV}$ protons at IHEP with efficiency reaching $85 \%$ at intensities well over $10^{12}$ particles/s, steered by silicon crystal as short as $2 \mathrm{~mm}$ [4]. A bent crystal ( $5 \mathrm{~mm} \mathrm{Si}$ ) is installed into the Yellow ring of the Relativistic Heavy Ion Collider where it channels $\mathrm{Au}$ ions and polarized protons of $100-250 \mathrm{GeV} / \mathrm{u}$ in the framework of a collimation experiment.

Carbon nanotubes are cylindrical molecules made of carbon atoms. Nanotubes can be manufactured of different diameters - from a fraction of a $\mathrm{nm}$ to a few microns, different lengths - from a micron up to a few millimeters, different materials - usually carbon but also others [5]. This makes nanotubes a very interesting object for channeling research.

The purpose of the present paper is to look at how the channeling technique could be used to make beams of very small emittance. As a potential application we consider a microbeam facility being developed at BNL [6] where a variety of beams from $\mathrm{Fe}^{+26}$ to protons of $0.1-3 \mathrm{GeV} / \mathrm{u}$ is needed with the beam size of $\sim 10 \mu \mathrm{m}$ at a target. A traditional approach to the creation of a microbeam would be a $\sim 20 \mu \mathrm{m}$-thin wire placed in a circulating beam and a set of microcollimators cutting out a small part of the scattered-beam phase space [6]. Here the weak points can be a low flux of scattered particles in the direction of the extraction line; primary and secondary particles scattered off the collimators may contaminate the microbeam.

If a channeling structure is used instead of wire, it can trap the incident particles and deliver them into a single

\footnotetext{
*Corresponding author.

Email address: biryukov@mx.ihep.su
}

PACS numbers: $61.85 .+\mathrm{p}, 41.85 .-\mathrm{p}, 87.56 .-\mathrm{v}, 85.35 . \mathrm{Kt}$

required direction (i.e., the extraction line) instead of scattering them all the way around. That may give a large gain in the microbeam flux. The rest of the system may be unchanged: the same set of collimators, etc. Further benefit is a low divergence of the channeled beam as set by channeling acceptance; that would reduce the need in collimation down the line and may reduce the emittance of the microbeam. Finally, the channeled beam would have well-defined sharp edges and contain solely primary particles. The open point is how to make a channeling structure as small as about the size of wire, $\sim 0.02 \mathrm{~mm}$, or smaller. Below we suggest two approaches, with crystals and with nanotubes.

\section{CRYSTAL MICROBEAM}

The first suggestion is to use a micron-sized structure on a surface of a single crystal; such structures are a welldeveloped technique [8]. The easy way to do it is to take a crystal plate, mask a strip $10 \mu \mathrm{m}$ (or $1 \mu \mathrm{m}$ ) wide on the surface, and etch the surface to a depth of 10 (or 1) $\mu \mathrm{m}$. That leaves a strip of 10 by $10 \mu \mathrm{m}$ (or 1 by $1 \mu \mathrm{m}$ ) on the surface; this strip can channel particles, thus forming a microbeam. In order to separate in the angle and space the beam channeled of the strip from the particles nearby (in the crystal bulk and outside), we suggest having a strip shorter than the substrate plate (Fig. 1), and bending the whole structure. That makes a perfect separation downstream.

While the size of the microbeam source is set by the strip size, the divergence in the direction of bending is set by the channeling angle, $\left(2 E_{c} / p \nu\right)^{1 / 2}$, where $E_{c}$ is the critical transverse energy for channeled particles and $p \nu$ is the particle's momentum times velocity per unit charge. In slightly bent $\mathrm{Si}(100)$ with $E_{c} \approx 5 \mathrm{eV}$, the $0.1-3 \mathrm{GeV}$ protons have a divergence of $0.05-0.2 \mathrm{mrad}$. For fully stripped ions of $\mathrm{Fe}^{+26}$ in the range of $0.1-1 \mathrm{GeV} / \mathrm{u}$, the 


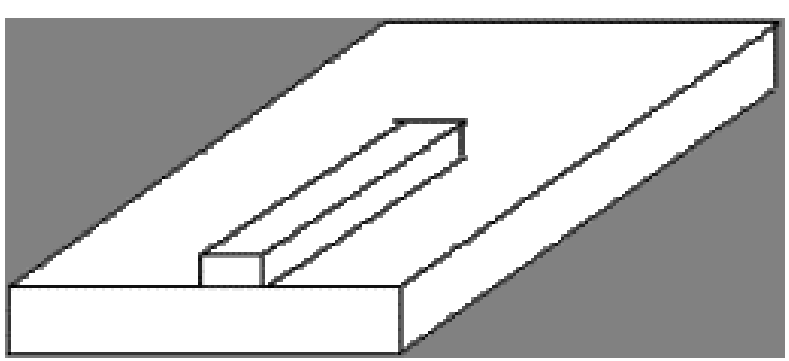

FIG. 1. Crystal with a micron-sized strip on the surface.

divergence is $0.08-0.15 \mathrm{mrad}$. One can pick crystal channels with bigger or smaller angular acceptance.

With a $1 \mu \mathrm{m}$ source, this gives a microbeam emittance of $(0.025-0.1) \pi \mathrm{nm}$ rad for protons of $0.1-3 \mathrm{GeV}$, and (0.04-0.08) $\pi \mathrm{nm} \mathrm{rad}$ for $\mathrm{Fe}^{+26}$ ions of $0.1-1 \mathrm{GeV} / \mathrm{u}$, in horizontal plane. For comparison, the horizontal emittance expected [6] from the traditional approach is $23 \pi \mathrm{nm} \mathrm{rad}$ at any energy. If realized, the channeling approach would give an improvement by a factor of $\approx$ 200-1000 for protons and 300-600 for ions. It can be improved even further by collimation downstream.

In the direction orthogonal to bending, microbeam divergence equals that of the circulating beam. However, the vertical size of the microbeam is set by the strip, down to $\sim 1 \mu \mathrm{m}$, while in a traditional approach it has to be cut by microcollimation. Therefore, an improvement of $\approx 100$ in vertical emittance can be expected from the channeling approach due to the small size of the source.

\section{NANOBEAM}

While crystal channeling is a well-established technique, nanotube channeling is just emerging as a beam instrument [9-13]. Although channeling effects can be observed in $0.3-1 \mathrm{~nm}$ wide cylindrical channels in the crystals of zeolite [14], where particles are confined within the potential well with geometry very similar to that of the nanotube, the channeling effect was not yet observed experimentally in nanotubes. Here, the beam can be trapped in a single nanotube cylinder of $\approx 1 \mathrm{~nm}$ diameter or in a rope consisting of many nanotubes. The depth $E_{c}$ of the potential well in a carbon nanotube is $\sim 15-60 \mathrm{eV}$ for channeled particles, depending on nanotube configuration [11]. The critical angle for channeling $\theta_{c}=\left(2 E_{c} / p \nu\right)^{1 / 2}$ is a factor of $\sim 1.5-3$ greater than with Si crystal. Provided that nanotubes can efficiently channel and deflect particle beams, they offer an interesting opportunity to make clean beams of a potentially very small size, down to 1 square nanometer if needed.

We have developed a Monte Carlo code and done simulations of particle channeling in bent single-wall nanotubes, aimed at finding out how useful nanotubes are for the channeling of positively charged particle beams, what kind of nanotubes are efficient for this job,

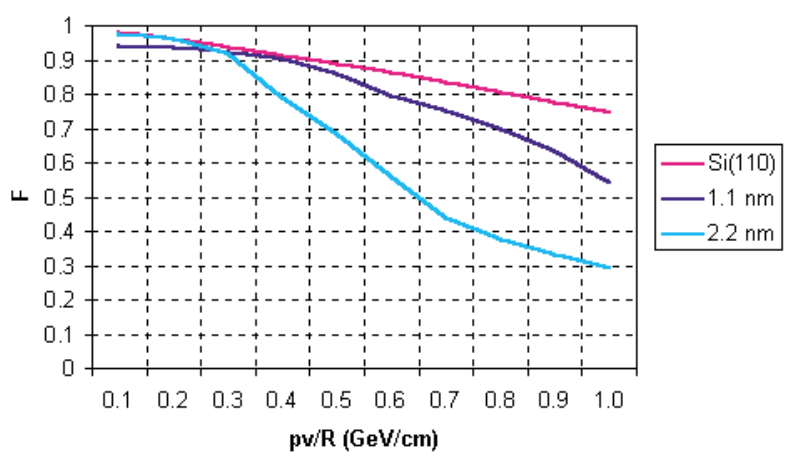

FIG. 2. (Color) The number of channeled protons as a function of the nanotube curvature $p \nu / R$ for tubes of different diameters (bottom up: 2.2 and $1.1 \mathrm{~nm}$ ). For comparison, also shown is the same function for $\mathrm{Si}(110)$ crystal (top curve).

and how nanotubes compare with crystals in this regard [12]. Figure 2 shows how the number of $1 \mathrm{GeV}$ protons channeled through a $50-\mu \mathrm{m}$-long nanotube depends on nanotube curvature $p \nu / R$ for tubes of different diameters (from Ref. [12]). For comparison, also shown is the same function for $\mathrm{Si}(110)$ crystal (from Ref. [1]). The channel length of $50 \mu \mathrm{m}$, with bending of $1 \mathrm{GeV} / \mathrm{cm}$, gives the $1 \mathrm{GeV}$ particles a deflection of $5 \mathrm{mrad}$ - sufficient for many accelerator applications such as extraction $[1,3,4,7]$. One can see from Fig. 2 that a nanotube as narrow as $1 \mathrm{~nm}$ is comparable to silicon crystal in beam bending.

For the simulations of nanotube channeling of $\mathrm{Fe}^{+26}$ ions and protons of $0.1-3 \mathrm{GeV} / \mathrm{u}$, we use the tubes of $1.1 \mathrm{~nm}$ diameter, typical for easily manufactured carbon nanotubes. We take the curvature radius of $2 \mathrm{~cm}$; then the beam energy range to be studied nearly corresponds to the $p \nu / R$ range studied in Fig. 2 . We choose the nanotube bending angle of $5 \mathrm{mrad}$. Figure 3 shows two examples of the angular distribution of protons downstream of the bent nanotube, shown in the direction of bending. Similar to pictures of bent crystal channeling, there is

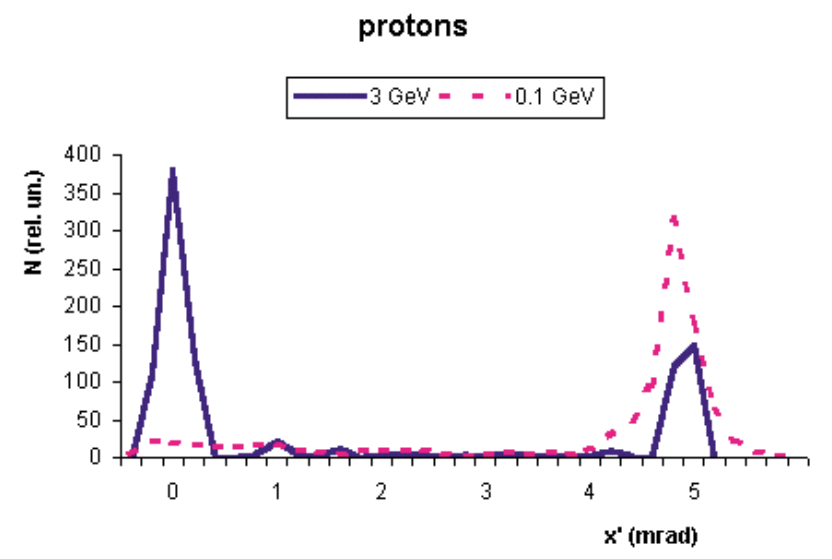

FIG. 3. (Color) The angular distribution of protons downstream of the bent nanotube, shown for two energies. 


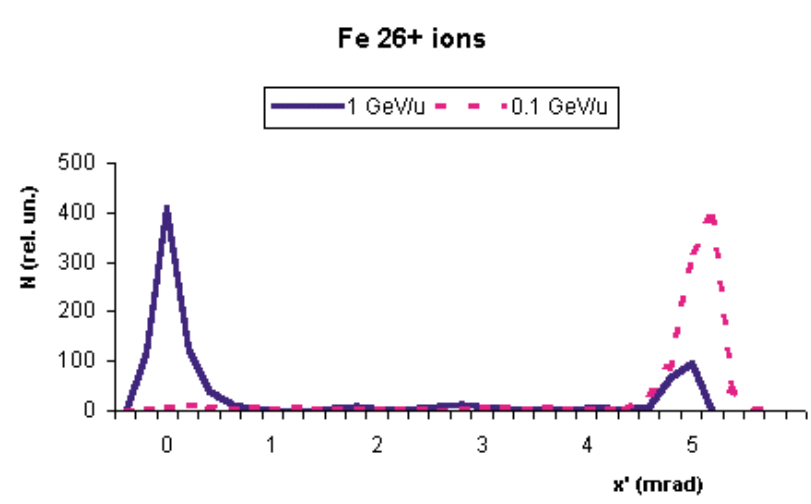

FIG. 4. (Color) The angular distribution of $\mathrm{Fe}^{+26}$ ions downstream of the bent nanotube, shown for two energies.

clear separation of channeled and nonchanneled peaks, with few particles lost (dechanneled along the tube) between them. For higher energy there is a substantial loss in efficiency due to centrifugal dechanneling, while for lower energy nearly all the particles trapped by the nanotube were channeled to its end. Overall, the transmission of particles by the tube is reasonably good on both ends of the energy range. The intermediate energies fall between the two cases shown.

The case of $\mathrm{Fe}^{+26}$ ions is shown in Fig. 4, again for both ends of the energy range of interest, 0.1 to $1.0 \mathrm{GeV}$ per nucleon. A similar picture can be seen, as with protons. Overall, for the similar ratio of beam momentum per unit charge, the angular distributions of $\mathrm{Fe}$ ions and protons are similar. The transmission efficiency is reasonably good for all particle species. The same nanotube deflector could be used in each case, throughout the range of energies and particle species.

For $0.1-1 \mathrm{GeV} / \mathrm{u}$ ions of $\mathrm{Fe}^{+26}$, the divergence of the channeled beam in a nanotube of arbitrary helicity such as $(11,9)$ is $0.24-0.77 \mathrm{mrad}$. The size of the source could be quite small. A typical nanorope (consisting of 1001000 nanotubes) would be a source that gives an emittance of the nanobeam of the order of $0.001 \pi \mathrm{nm}$ rad both horizontally and vertically, factor of 10000 down from the figure potentially achievable with a "traditional amorphous" source.

\section{INTENSITY OF MICROBEAM}

With small emittance, microbeam intensity is also small. However, the applications such as a microbeam facility [6] require quite small intensities, down to 1-1000 particles/s. With some $10^{9}-10^{11}$ particles stored in the AGS ring, this gives enough room for constructing beams of very small emittances discussed above.

Let us take the example of AGS to estimate the achievable intensity of the channeled microbeam. The beam circulating in the AGS ring has the size about $\pm 5 \mathrm{~mm}$ before the extraction septum. The beam store is typically
$10^{9}$ ions or $10^{11}$ protons. An area of $1 \mu \mathrm{m}^{2}$ would be hit by $\sim 10$ ions (or 1000 protons) in the time of a single turn in the ring ( $\sim 1 \mu \mathrm{s}$ at $1 \mathrm{GeV}$ per nucleon); the hit rate is then $\sim 10^{7}$ ions/s per $1 \mu \mathrm{m}^{2}$.

The divergence of particles incident at crystal in the periphery of the circulating beam, after crossing a stripping foil, is expected to be several times bigger than channeling acceptance. For particles trapped by a crystal or nanotube, the transmission factor would be $10 \%$ to $100 \%$ (e.g., Figs. 3 and 4) if channeled particles are bent a few mrad.

Microbeam intensity of $10^{5}-10^{7}$ ions/s appears even far greater than needed (though it is easily reduced by moving the crystal away from the core of the beam or misaligning it). One can put the question differently: how much a crystal can survive? The IHEP experience shows that crystals can channel up to $\sim 3 \times 10^{12}$ particles/s per cross section of $0.5 \times 5 \mathrm{~mm}^{2}$ without cooling measures. This corresponds to $10^{6} /\left(\mathrm{s} \mu \mathrm{m}^{2}\right)=1 /\left(\mathrm{s} \mathrm{nm}^{2}\right)$. So, a microcrystal structure can channel much more particles than needed, and even a nanorope could do the job.

A lifetime of $\sim 5 \times 10^{20}$ proton irradiation per $\mathrm{cm}^{2}$ as measured [15] for channeling crystal corresponds to $5 \times$ $10^{12} / \mu \mathrm{m}^{2}$; this means over 100 years of operation of $1 \mu \mathrm{m}^{2}$ crystal with channeling of $\sim 1000$ protons/s, or one year for $(20 \mathrm{~nm})^{2}$ nanorope operating at 100 protons/s.

\section{CONCLUSION}

The use of the microcrystal/nanotube channeling technique allows extracted beams of a very small size. That would drastically improve the precision of tumor therapy with ion beams and allow to handle delicate cases of eye and brain tumors at medical accelerators, make possible a selective irradiation ("surgery") of cells [6] or smaller biological objects, may be convenient for calibration of microdetectors, and offer new opportunities for high energy physics experiments and industrial applications.

\section{ACKNOWLEDGMENTS}

The authors thank K. A. Brown for discussions and R. P. Fliller for comments. This work was partially supported by INFN - Gruppo V, as a NANO experiment, and by INTAS-CERN Grant No. 132-2000.

[1] V. M. Biryukov, Yu. A. Chesnokov, and V. I. Kotov, Crystal Channeling and its Application at High Energy Accelerators (Springer, Berlin, 1997). See also http:// crystalbeam.narod.ru

[2] M. B. H. Breese, Nucl. Instrum. Methods Phys. Res., Sect. B 132, 540 (1997).

[3] R. A. Carrigan, Jr. et al., Phys. Rev. ST Accel. Beams 1, 022801 (1998); R. A. Carrigan, Jr. et al., Phys. Rev. ST Accel. Beams 5, 043501 (2002). 
[4] A. G. Afonin et al., Phys. Rev. Lett. 87, 094802 (2001).

[5] T.W. Ebbesen, Phys. Today 49, No. 6, 26 (1996); Z. Y. Wu et al., Appl. Phys. Lett. 80, 2973 (2002).

[6] K. A. Brown et al., in Proceedings of the EPAC 2002, Paris (CERN, Geneva, 2002), p. 554.

[7] R. P. Fliller III et al., in Proceedings of the EPAC 2002, Paris (Ref. [6]), p. 200.

[8] P. Kleimann, J. Linnros, and R. Juhasz, Appl. Phys. Lett. 79, 1727 (2001).

[9] V.V. Klimov and V.S. Letokhov, Phys. Lett. A 222, 424 (1996).
[10] L. G. Gevorgian, K. A. Ispirian, R. K. Ispirian, JETP Lett. 66, 322 (1997).

[11] N. K. Zhevago and V. I. Glebov, Phys. Lett. A 250, 360 (1998).

[12] V. M. Biryukov and S. Bellucci, Phys. Lett. B 542, 111 (2002).

[13] S. Bellucci et al., Nucl. Instrum. Methods Phys. Res., Sect. B 202, 236 (2003).

[14] R. Kirsch and X. Artru (private communication).

[15] A. Baurichter et al., Nucl. Instrum. Methods Phys. Res., Sect. B 164-165, 27 (2000). 